\title{
Global Synthesis Method for the Optimization of Multifeed EBG Antennas
}

\author{
Julien Drouet, Marc Thevenot, Régis Chantalat, Cyrille Menudier, Majed Koubeissi, \\ Thierry Monédière, and Bernard Jecko
}

XLIM Laboratory, University of Limoges, Limoges 87060, France

Correspondence should be addressed to Marc Thevenot, marc.thevenot@xlim.fr

Received 19 March 2008; Revised 9 July 2008; Accepted 15 August 2008

Recommended by Karu Esselle

This paper presents a novel technique for synthesizing a given radiation pattern from an EBG antenna with an array feed. The method determines the optimum sets of input waves and input impedances for the feed ports in order to perform simultaneously the radiation pattern and the impedance matching of all the radiating probes that form the array feed. The method is validated through a numerical design of an EBG antenna excited with four patch antennas. The structure is designed to radiate with a single lobe scanned at $\theta=30^{\circ}$ in the E-plane. The interactions between each patch inside the EBG resonator are characterized with the CST MWS software. The optimum weights and the input impedances which simultaneously perform the objective radiation and the matching of all feeding ports are calculated by the developed global synthesis method. The feed network is designed with the Agilent ADS software in order to perform the specified weights and the impedances matching.

Copyright (c) 2008 Julien Drouet et al. This is an open access article distributed under the Creative Commons Attribution License, which permits unrestricted use, distribution, and reproduction in any medium, provided the original work is properly cited.

\section{INTRODUCTION}

The electromagnetic band gap (EBG) antennas have been extensively studied over the last decade. Their functioning principle is as the leaky wave antennas [1-4]. A partially reflective wall (PRW), an EBG material [5-7], or a frequency selective surface (FSS) [8-11], for instance, lies above a metallic ground plane in order to build a planar waveguide. The first electromagnetic waveguide mode can radiate through the PRW, while its phase velocity is greater than the light's one, in other words, when the operating frequency is close to the cutoff of the waveguide mode. This leaky mode is excited with a radiating feed (like a patch antenna) or a set of several feeds (like an array of small antennas [12-17]). The resulting radiation pattern of the whole EBG structure depends on the radiation of the feeds. The excitation of the EBG antenna with a feed array is useful for the next two reasons:

(i) several feeds can be distributed under the PRW to enhance the directivity without reducing the bandwidth [12]; the distance between the feeds can be enlarged beyond one half wavelength because the EBG material is efficient to degrade the grating lobes [13]; thus, it is relatively easy to reach high directivities with few probes; this can simplify the design of the feed network;

(ii) an array antenna with few feeds can be used to select a steered angle; the radiations of the different feeds under the EBG PRW are reinforced in a desired direction and suppressed in undesired directions, while the EBG structure purpose is to enhance the gain in this desired direction [14-17].

The functioning of an array feed for an EBG resonator seems different from the functioning of an usual phased array antenna [18-21]. Since the radiating probes lie between the ground plane and the PRW, the waveguide mode interacts strongly with them. Consequently, their input impedances are different from the initial impedances found without the PRW, the mutual couplings could be ten times greater than without the PRW. Moreover, their radiation patterns are strongly conditioned by the interactions with both the EBG resonator and the others nearby probes. So, if no cares are taken when designing, the strong mutual couplings between the probes can damage the functioning of the feed network.

Thus, a rigorous technique must be used to design the array feed for the EBG antennas. It is described in Section 2 
of the paper. The aim is to realize simultaneously an objective radiation pattern and the greater radiation efficiency for a system made up of a multifeed EBG antenna and its feed network.

In Section 3, the technique is illustrated through the numerical studies of an EBG structure excited by four patch antennas. The whole EBG antenna has been designed to radiate in the direction $\theta=30^{\circ}$ in the E-plane [4].

\section{DESCRIPTION OF THE GLOBAL SYNTHESIS METHOD}

This section explains the method that we have used for designing a multifeed EBG antenna made up of an array feed and a feed network. The problem is the optimization of this feed network by taking into account the strong couplings between the output ports of the array feed. The design technique is broken down into four steps. (1) The method requires a full-wave analysis (e.g., CST Microwave studio) to characterize the interactions between each probe inside the EBG resonator. During this analysis, each radiating probe is successively supplied in order to extract the antenna's $\mathbf{S}$ matrix and the radiation pattern of each probe. (2) The objective radiation pattern is synthesized by a linear combination of the probes' radiation patterns. (3) The optimum input impedances and the weights which simultaneously perform the objective radiation and the matching of all the probes are calculated by taking into account the strong interactions between the radiating probes. (4) Finally, the feed network can be designed to meet these specifications.

\subsection{Full-wave analysis of the EBG antenna}

This first step is a full-wave analysis of the EBG antenna with the array feed. During this analysis, each probe of the array feed is connected to an individual port in order to characterize the interactions between the probes and the EBG structure. The impedances of the port are annotated $\mathbf{Z g}$. The probes are successively supplied with a normalized incident wave $\mathbf{a}$ in order to extract their own radiation pattern called "active element pattern" [20] and the scattering matrix [Sc]. The active element patterns $\left(\boldsymbol{\Phi}_{i}\right)$ are vectors containing the electromagnetic fields $\left(E_{i}(\theta \times \varphi)\right)$.

\subsection{Synthesis of the objective radiation pattern}

The objective radiation pattern must be fit by a linear combination of the different "active element patterns." This second step consists to find the complex coefficients (or weight) for the linear combination. The objective radiation pattern $\left(\boldsymbol{\Phi}_{\mathrm{obj}}\right)$ can efficiently be formed by combining an array factor with the transfer function of the EBG material [15-17]. This linear combination is given by (1). The coefficients $\beta_{1, \ldots, N}$ are the elements of the weighting vector. A common matrix left division (computed by Gaussian

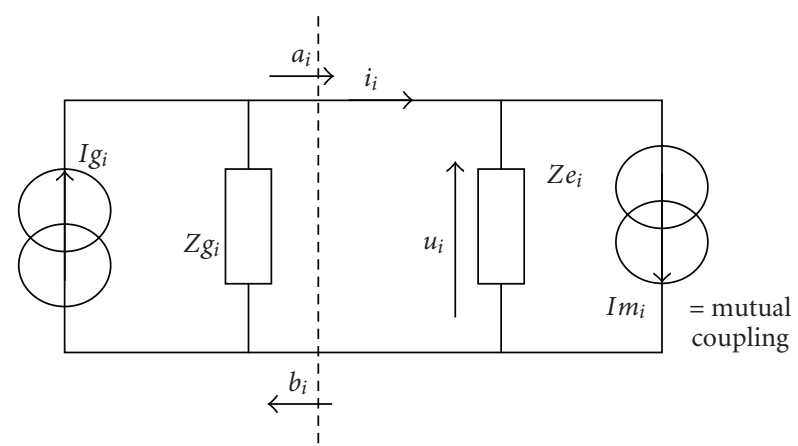

Figure 1: Antenna “ $i$ ” connected to a port of impedance $Z g_{i}$.

elimination) can be used to deduce the best combination of the $N$ radiation patterns (the $\beta$ vector):

$$
\underbrace{\left[\begin{array}{llll}
\boldsymbol{\Phi}_{1} & \boldsymbol{\Phi}_{2} & \cdots & \boldsymbol{\Phi}_{N}
\end{array}\right]}_{\left(\theta^{*} \varphi ; N\right)} \cdot\left(\begin{array}{c}
\beta_{1} \\
\beta_{2} \\
\vdots \\
\beta_{N}
\end{array}\right) \approx \underbrace{\left(\boldsymbol{\Phi}_{\mathrm{obj}}\right)}_{\left(\theta^{*} \varphi\right)} .
$$

\subsection{Definition of the specifications for the feed network}

The global synthesis method provides the specifications for the feeding ports. The aim is to optimize the radiation efficiency while maintaining the objective radiation function. These specifications are the normalized incident wave and the input impedance that must be connected to each port of the multifeed EBG antenna.

Figure 1 is a schematic representation of one radiating probe of the array feed. It is connected to a $Z g_{i}$ impedance port. In this figure, $Z e_{i}$ is the intrinsic impedance of the probe, and $I m_{i}$ is a current resulting from the mutual coupling with the other radiating probes. The current and the voltage in each probe are evaluated. According to the superposition theorem, the driven voltages $u_{i}$, which must excite each probe, are derived from (2). Let us notice that this equation takes into account the mutual coupling between the probes:

$$
(u)=(\operatorname{diag}(\sqrt{Z g}) \cdot[I+S c] \cdot \operatorname{diag}(a)) \cdot(\beta) .
$$

Vector $(a)$ and vector $(Z g)$ are, respectively, the waves that are used to supply each probe during the electromagnetic analysis of the EBG structure (first step) and the impedances of the ports.

The currents into the ports are deduced from (3):

$$
\begin{gathered}
(i)=[\alpha] \cdot(u) \\
{[\alpha]=\left[\operatorname{diag}\left(\frac{1}{\sqrt{Z g}}\right) \cdot[I-S c] \cdot \operatorname{diag}(a)\right]} \\
\cdots \cdot \operatorname{diag}(\sqrt{Z g}) \cdot[I+S c] \cdot \operatorname{diag}(a)]^{-1}
\end{gathered}
$$


As for the currents, the input impedances depend on both the coupling matrix Sc and the voltages that are needed to supply the probes. They are the elements of a scan impedance matrix $\left(Z_{\text {scan }}\right)$ which is given by $(6)$ :

$$
Z_{\text {scan }}=u \cdot /([\alpha] \cdot u) .
$$

According to (3) and (4), (5) becomes

$$
\begin{aligned}
Z_{\text {scan }} & =([\operatorname{diag}(\sqrt{Z g}) \cdot[I+S c] \cdot \operatorname{diag}(a)] \cdot \beta) \cdot / \\
& \cdots([\operatorname{diag}(1 / \sqrt{Z g}) \cdot[I-S c] \cdot \operatorname{diag}(a)] \cdot \beta) .
\end{aligned}
$$

\subsection{Design of the feed network}

In order to maximize the radiation efficiency, the ports of the feed network must match the $Z_{\text {scan }}$ impedances. Each port " $i$ " of the feed network must excite the ports of the EBG antenna with the incident waves specified by the relation (7) because the $Z_{\text {scan }}$ impedances are achieved only when the relation (2) is fulfilled:

$$
a_{i}=u_{i} \frac{\sqrt{\operatorname{Re}\left(Z_{\text {scan }}\right)}}{Z_{\text {scan }}} .
$$

When these conditions (6), (7) will be fulfilled by the feed ports, the coupled power does not disturb the functioning of the feed network. So, the whole incident power is transmitted to the probes, the radiation efficiency is maximized and the weighting vector $(\beta)$ is realized.

\section{NUMERICAL VALIDATION}

In this section, a proof is given to how useful and efficient the global synthesis method is when designing EBG antennas. For this purpose, the following studies will focus on the design of a leaky wave EBG antenna which is excited by four radiating patches.

The PRW is a $2.466 \mathrm{~mm}$ thick alumina slab with a permittivity of 9.4. It lies $15.0 \mathrm{~mm}$ above the ground plane. The EBG structure is designed to enhance the directivity in the direction $\theta=30^{\circ}$ at the frequency $f_{0}=11.35 \mathrm{GHz}$ $[4,15-17]$. The global EBG antenna dimensions are $L x=$ $330 \mathrm{~mm}$ and $L y=280 \mathrm{~mm}$. The antenna is widely oversized in order to neglect the edge effects. The four patches are organized into a $2 \times 2$ array (Figure 2 ) and spaced out by $13.2 \mathrm{~mm}(0.5 \lambda 0)$. They are printed on a $0.5 \mathrm{~mm}$ thick Teflon $(\varepsilon r=2.08)$ substrate. The microstrip feed network which has to generate the weights and the complex conjugate of the patch impedances must not interfere with the antenna. We have chosen to print it behind the antenna ground plane onto another Teflon substrate. Four $50 \Omega$ coaxial transitions are drilled through the ground plane and used to connect the microstrip ports to the patch antennas (Figure 3 ).

An evaluation of the ability for the EBG antenna to radiate with a single lobe in the direction $\theta=30^{\circ}$ was first made with the analytical method described in $[4,8,15-$ 17]. This analysis provides a theoretical radiation pattern that can constitute the objective one $\left(\boldsymbol{\Phi}_{\text {obj }}\right)$. The EBG spatial filter enhances the radiations nearly the $\theta=30^{\circ}$ direction

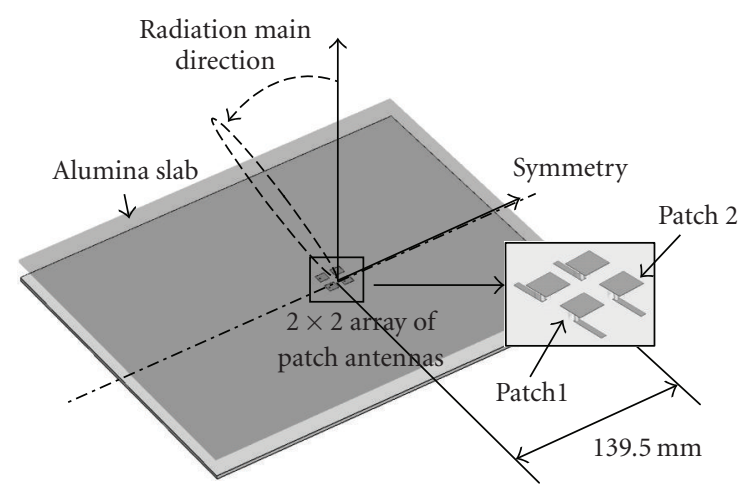

FIGURE 2: The EBG leaky wave antenna is fed with a patch array. It must radiate in the direction $\left[\theta=30^{\circ} ; \varphi=0^{\circ}\right]$.

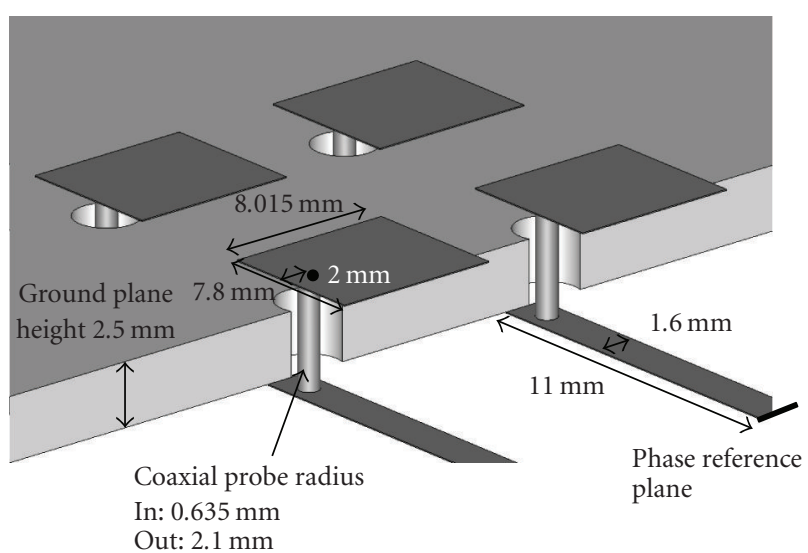

FIgURE 3: The patches are printed onto a Teflon substrate. They are fed through the ground plane by a $50 \Omega$ coaxial probe. A second Teflon substrate is set onto the back face of the metallic plane. It receives the printed feed network.

whatever the $\varphi$ angle $[4,14-17]$. The patch array is then weighted in order to eliminate the radiation in the opposite direction $\left(\left\{\theta=30^{\circ} ; \varphi=180^{\circ}\right\}\right.$ or $\left.\left\{\theta=-30^{\circ} ; \varphi=0^{\circ}\right\}\right)$. The radiation pattern of the multifeed EBG antenna is estimated by multiplying the EBG spatial filtering function with the patch array radiation pattern. The patch array radiation pattern, the spatial filtering function, and their product are plotted in Figure 4. The weights, which are applied to the patches in order to obtain the objective radiation, are written in Table 1. Only two weights appear in the table because the structure is symmetric. It should to be stressed that these results are approximated since the analysis considers an infinite dimensional EBG structure and the patch antennas do not interfere with each other. The radiation pattern illustrated by the black solid line in Figure 4 can be used as the objective radiation pattern $\Phi_{\text {obj }}$ (1).

In the next step, we have used CST Microwave studio to achieve the full-wave analysis of the whole EBG structure. Thanks to the symmetry, only two patches among the four are supplied consecutively in order to obtain their own radiation patterns. These "active element patterns" are 


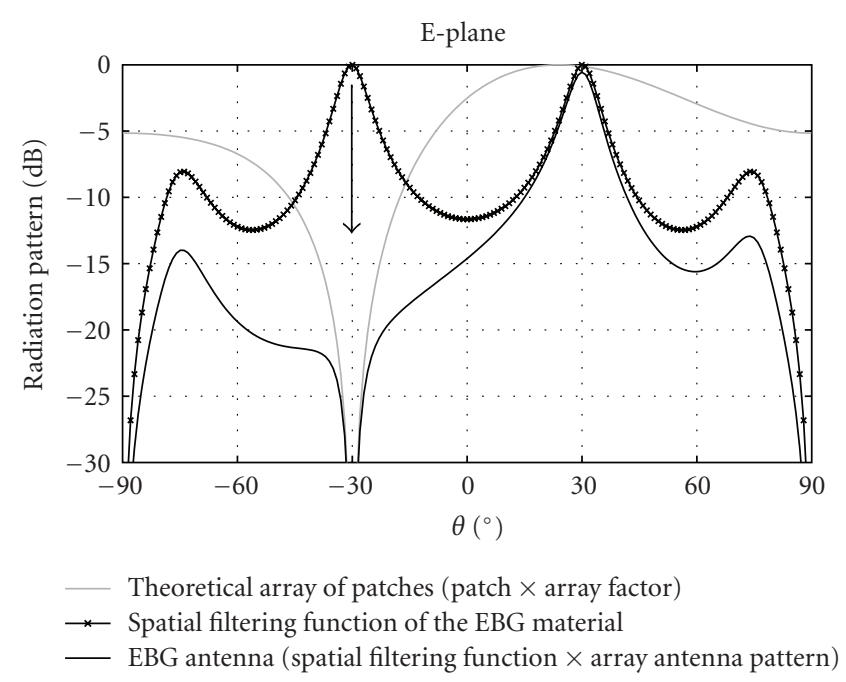

FIgURE 4: The patch array $(2 \times 2$ patches $)$ selects a radiation direction within the conical beam steering.

TABLE 1: Theoretical array factor.

\begin{tabular}{lcc}
\hline Weights & Patch 1 & Patch 2 \\
\hline Magnitude & 1 & 1 \\
Phase & $0^{\circ}$ & $90^{\circ}$ \\
\hline
\end{tabular}

TABLE 2: Weight that may be assigned to the radiation patterns of active element at $11.35 \mathrm{GHz}$.

\begin{tabular}{lcc}
\hline$\beta$ & $\beta_{1}($ patch 1$)$ & $\beta_{2}($ patch 2$)$ \\
\hline Magnitude & 1 & 0.585 \\
Phase & $0^{\circ}$ & $73^{\circ}$ \\
\hline
\end{tabular}

plotted in Figure 5. According to (1), the weights $\beta_{1}$ and $\beta_{2}$ are deduced and written in Table 2. Thus, Figure 6 points out the resemblance between the objective radiation pattern and the linear combination of the active element patterns weighted by the coefficients $\beta_{1}$ and $\beta_{2}$.

Figure 7 shows the scattering matrix of the patch array with (right) and without (left) the PRW above the ground plane. The comparison shows that the mutual coupling between the patches is dramatically increased (about $10 \mathrm{~dB}$ ) by the interactions with the EBG structure. These interactions should not be omitted when connecting the array feed with the feed network.

The optimum weights and the input impedances which simultaneously perform the objective radiation and the matching of all the feeding ports can be calculated. The driven voltages $\left(u_{1}, u_{2}\right)$, the impedances $\left(Z_{\text {scan } 1}, Z_{\text {scan } 2}\right)$, and the weights $\left(a_{1}, a_{2}\right)$ are obtained from (5), (6), and (7), the scattering matrix and $\left(\beta_{1}, \beta_{2}\right)$. They are shown in Table 3 . Even though the patch antennas seem well matched to $50 \Omega$ when they are successively fed (Figure 7), it appears that the impedances that optimize the efficiency are strongly affected by the mutual couplings. They are quite different from $50 \Omega$.

The design of the microstrip feed network has been made with the Agilent ADS (Momentum) software in order to

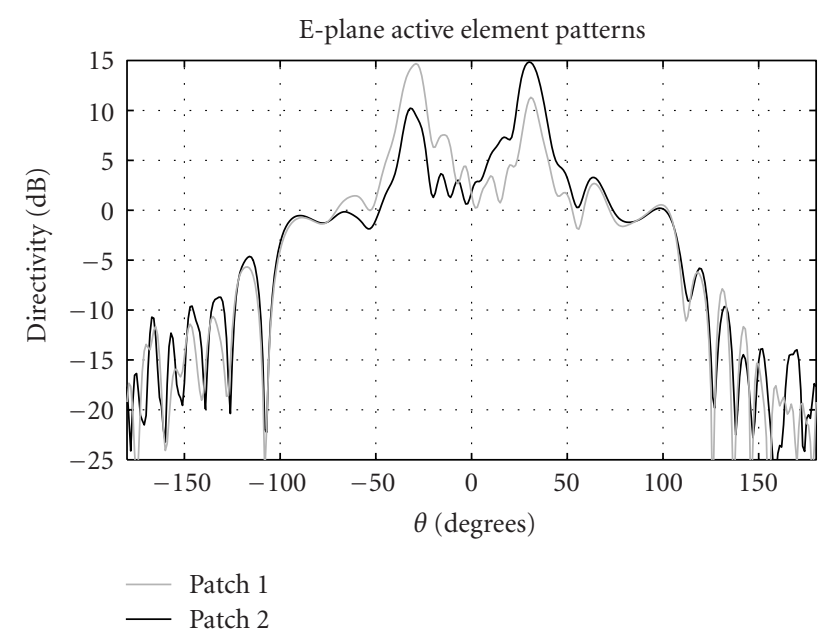

FIgUre 5: Active element patterns [20] when the EBG structure is successively fed by the patches 1 and 2 (E-plane: $11.35 \mathrm{GHz}$ ).

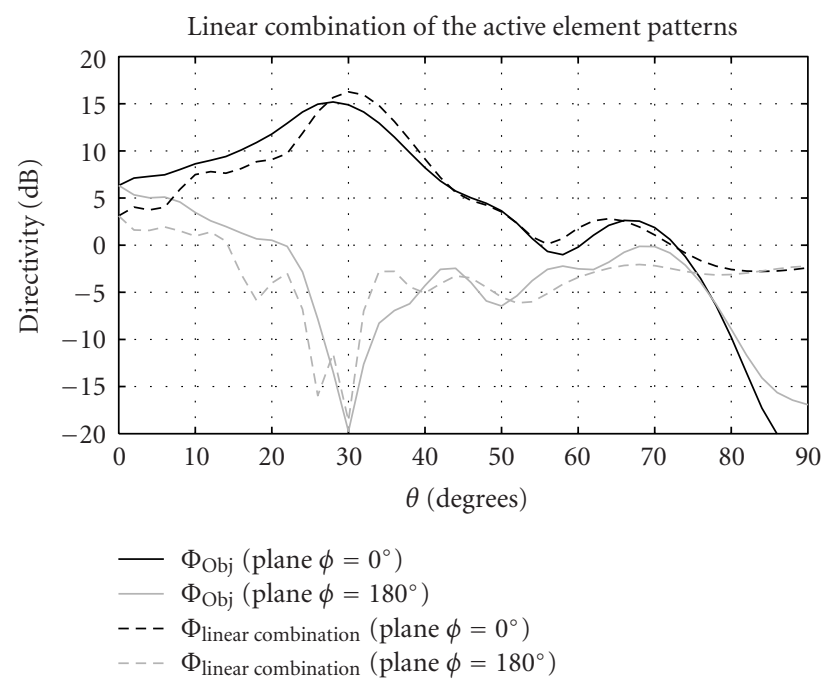

Figure 6: Comparison of the objective radiation pattern with the linear combination of the active element patterns (E-Plane: $11.35 \mathrm{GHz})$.

perform the weights and the impedance matching specified by the global synthesis method (Table 3 ). When designing the feed network, the impedances of the ports are set equal to the impedances $Z_{\text {scan }}$. Due to the magnetic symmetry, only one arm of the network is shown (Figure 8).

We consider two validations for the global design with CST. First, the scattering matrix of the feed network and the EBG antenna are numerically connected together. The radiation pattern and the return loss are computed. These results are then compared with an entire structure simulation. For this entire full-wave simulation, the feed network and the EBG antenna are meshed under an unique computational volume. Figure 9 plots the radiation patterns in the E-plane. The two validations (entire structure simulation and the $S$ matrix connection) are compared with the objective linear 


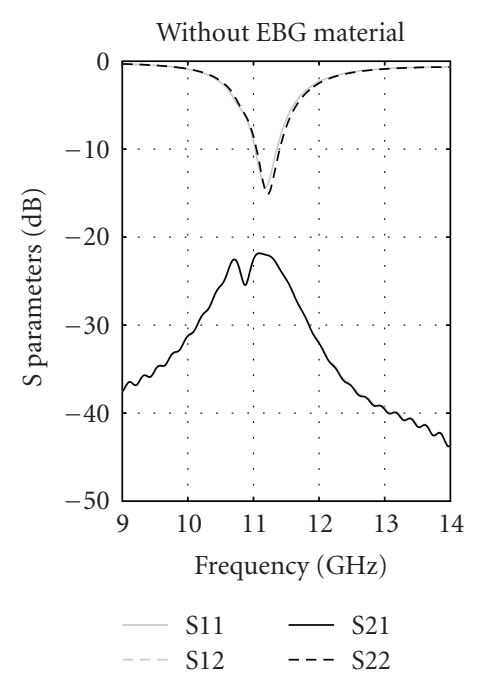

(a)

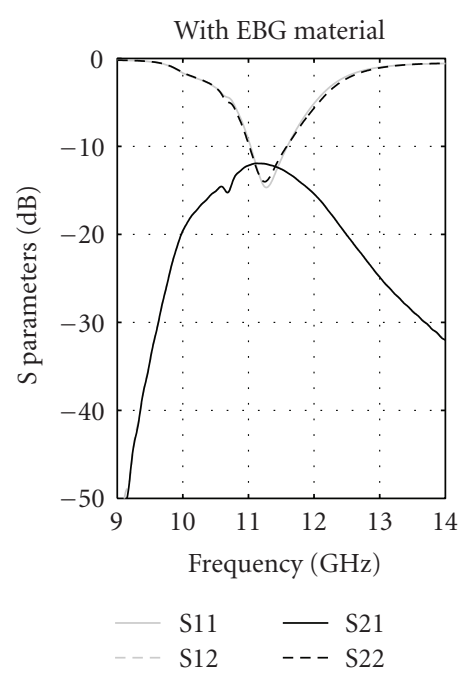

(b)

FIGURE 7: $S$ parameters of the array antenna $(2 \times 2$ patches $)$ and $S$ parameters of the same array antenna inside the EBG structure.

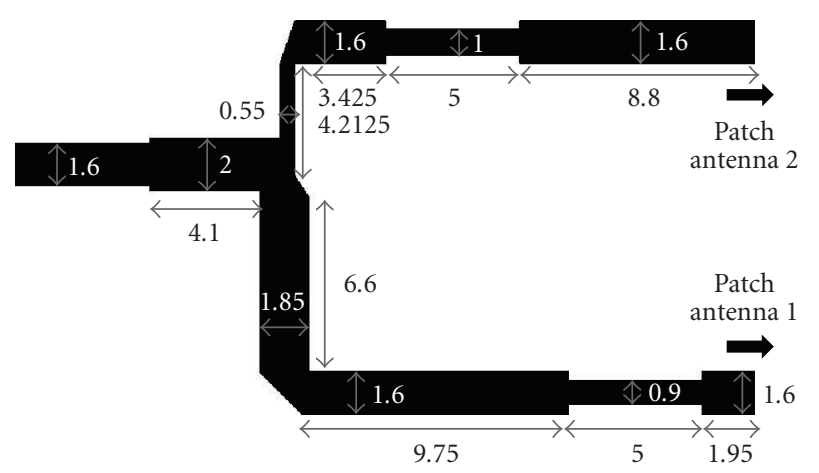

FIGURE 8: The feed network is matched to impedances $Z_{\text {scan_l } 1 / 2}$ and it achieves the weights shown in Table 3.

TABLE 3: Electrical characteristics of the patches array, $F=$ $11.35 \mathrm{GHz}$.

\begin{tabular}{cccc}
\hline \multicolumn{2}{c}{ Weights } & Patch 1 & Patch 2 \\
\hline \multirow{2}{*}{ Voltage $U_{i}$} & Magnitude & 1 & 0.7263 \\
& Phase & $0^{\circ}$ & $50.3^{\circ}$ \\
\hline \multicolumn{2}{c}{ Impedances $Z_{\text {scan }}$} & $50.7+25.6 \mathrm{j} \Omega$ & $83.7-47.5 \mathrm{j} \Omega$ \\
\hline \multirow{2}{*}{$a_{i}$} & Magnitude & 0.876 & 0.482 \\
& Phase & $0^{\circ}$ & $106^{\circ}$ \\
\hline
\end{tabular}

combination of the active element patterns $\left(\beta_{1} \cdot \phi_{1}(\theta, \varphi)+\right.$ $\beta_{2} \cdot \phi_{2}(\theta, \varphi)$ ). It is obvious that the two validations agree well with the objective radiation pattern. We can notice a minor difference in the backward directions for the entire structure simulation. It is probably caused by the direct radiation of the feed network. The return loss is plotted in Figure 10. The level is lower than $-25 \mathrm{~dB}$ at the optimization frequency $(11.35 \mathrm{GHz})$ for the two validations and it stay lower than $-15 \mathrm{~dB}$ over a wide-frequency band.

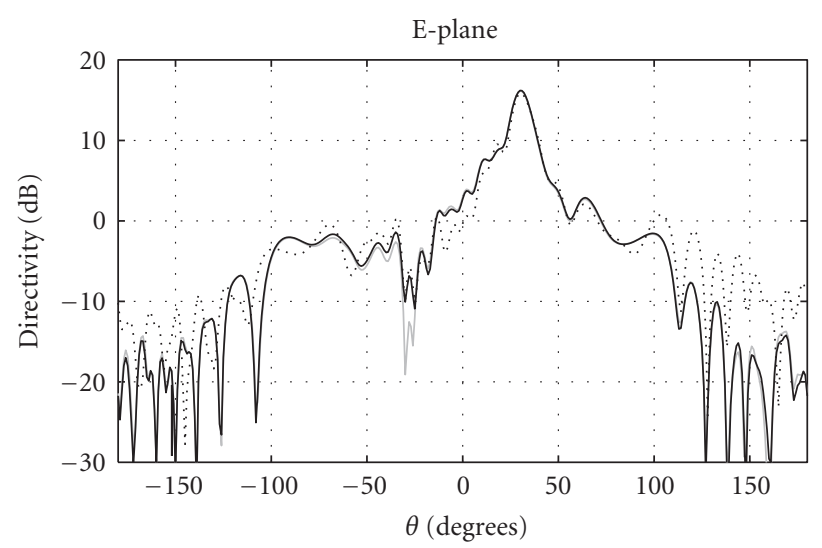

- Linear combination of the active element patterns

- Connection of the feed network $S$ matrix with the EBG antennas ..... Entire structure simulation

FIGURE 9: Radiation pattern comparison in the E-plane. 3 radiation patterns are plotted. The first plot is obtained by the linear combination of the active element patterns; the second is CST MWS posttreatment taking into account the feed network scattering matrix; and the third plot results from an entire structure simulation (the feed network and the EBG antenna are meshed under the same CST simulation).

The numerical validations show that the radiation pattern is successfully synthesized as well as the impedance matching of every port. Through the precision of our results, we demonstrate that the method is very efficient for synthesizing a given radiation pattern from an EBG antenna with an array feed.

\section{CONCLUSION}

This paper illustrates the usefulness and the efficiency of using a global synthesis method when designing an array 


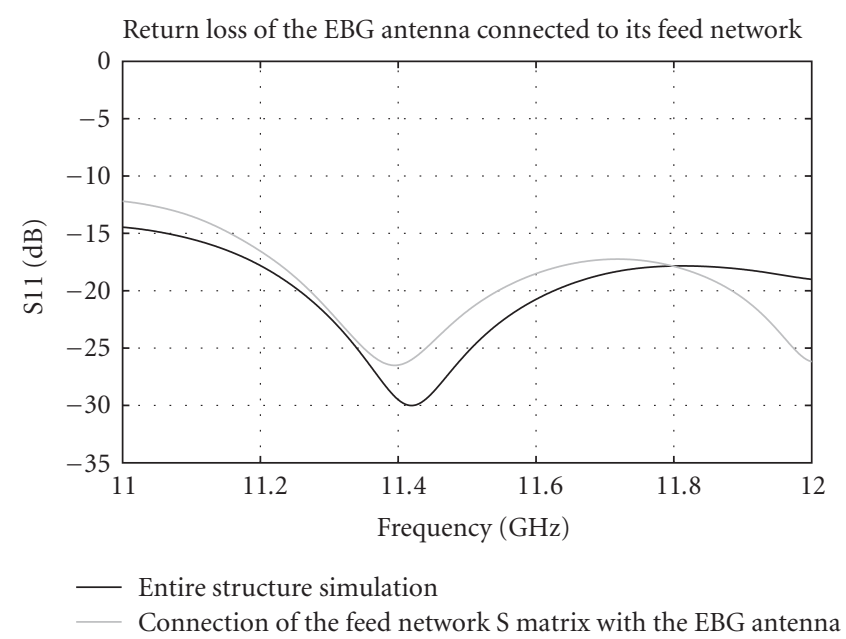

FIgURE 10: Return loss of the antenna. S11 is calculated by connecting the antenna with the feed network scattering matrix (grey bold line). This S11 is compared with the S11 computed when the antenna and its feed network are meshed under the same CST MWS simulation (black line).

feed for an EBG antenna. In such structures, the radiating probes strongly interact with each other and with the EBG material. The intensity of the coupled waves may damage the functioning of a feed network if no cares are taken. The global synthesis method provides the input impedances and the weights for the input waves that optimize the efficiency of the antenna, while an objective radiation pattern is accomplished. To sum up, the feed network can simply be designed with momentum, while the used port impedances are taken as the optimization impedances of the array feed for the EBG antenna.

\section{REFERENCES}

[1] N. G. Alexopoulos and D. R. Jackson, "Fundamental superstrate (cover) effects on printed circuit antennas," IEEE Transactions on Antennas and Propagation, vol. 32, no. 8, pp. 807-816, 1984.

[2] D. R. Jackson and N. G. Alexopoulos, "Gain enhancement methods for printed circuit antennas," IEEE Transactions on Antennas and Propagation, vol. 33, no. 9, pp. 976-987, 1985.

[3] H. Y. Yang and N. G. Alexopoulos, "Gain enhancement methods for printed circuit antennas through multiple superstrates," IEEE Transactions on Antennas and Propagation, vol. 35, no. 7, pp. 860-863, 1987.

[4] D. R. Jackson, A. A. Oliner, and A. Ip, "Leaky-wave propagation and radiation for a narrow-beam multiple-layer dielectric structure," IEEE Transactions on Antennas and Propagation, vol. 41, no. 3, pp. 344-348, 1993.

[5] M. Thevenot, C. Cheype, A. Reineix, and B. Jecko, "Directive photonic-bandgap antennas," IEEE Transactions on Microwave Theory and Techniques, vol. 47, no. 11, pp. 2115-2122, 1999.

[6] M. Qiu and S. He, "High-directivity patch antenna with both photonic bandgap substrate and photonic bandgap cover," Microwave and Optical Technology Letters, vol. 30, no. 1, pp. 41-44, 2001.
[7] T. Akalin, J. Danglot, O. Vanbésien, and D. Lippens, "A highly directive dipole antenna embedded in a Fabry-Perot type cavity," IEEE Microwave and Wireless Components Letters, vol. 12, no. 2, pp. 48-50, 2002.

[8] G. V. Trentini, "Partially reflecting sheet arrays," IRE Transactions on Antennas and Propagation, vol. 4, no. 4, pp. 666-671, 1956.

[9] A. P. Feresidis and J. C. Vardaxoglou, "High gain planar antenna using optimised partially reflective surfaces," IEE Proceedings: Microwaves, Antennas and Propagation, vol. 148, no. 6, pp. 345-350, 2001.

[10] R. Sauleau, "Fabry Perot resonators," in Encyclopedia of RF and Microwave Engineering, K. Chang, Ed., vol. 2, pp. 1381-1401, John Willey \& Sons, New York, NY, USA, 2005.

[11] P. Kosmas, A. P. Feresidis, and G. Goussetis, "Periodic FDTD analysis of a 2-D leaky-wave planar antenna based on dipole frequency selective surfaces," IEEE Transactions on Antennas and Propagation, vol. 55, no. 7, pp. 2006-2012, 2007.

[12] L. Leger, T. Monédière, and B. Jecko, "Enhancement of gain and radiation bandwidth for a planar 1-D EBG antenna," IEEE Microwave and Wireless Components Letters, vol. 15, no. 9, pp. 573-575, 2005.

[13] R. Gardelli, M. Albani, and F. Capolino, "Array thinning by using antennas in a Fabry-Perot cavity for gain enhancement," IEEE Transactions on Antennas and Propagation, vol. 54, no. 7, pp. 1979-1990, 2006.

[14] H. Boutayeb, K. Mahdjoubi, and A.-C. Tarot, "Frequency beam scanning and gain enhancement properties of PBG antennas," in Proceedings of the IEEE Antennas and Propagation Society International Symposium, p. 526, Columbus, Ohio, USA, June 2003.

[15] J. Drouet, M. Thevenot, T . Monédière, and B. Jecko, "Analytical method for EBG resonator antennas design, FDTD validation," in Proceedings of the 1st European Conference on Antennas and Propagation (EuCAP'06), pp. 1-5, Nice, France, November 2006, paper ID 346557.

[16] M. Thevenot, J. Drouet, B. Jecko, et al., "New advancements to exploit the potentialities of the EBG resonator antennas," in Proceedings of the IEEE Antennas and Propagation Society International Symposium, vol. 3A, pp. 22-25, Washington, DC, USA, July 2005.

[17] L. Leger, T. Monédière, M. Thevenot, and B. Jecko, "Multifrequency and beam steered electromagnetic band gap antennas," in Proceedings of the IEEE Antennas and Propagation Society International Symposium, vol. 2, pp. 1151-1154, Monterey, Calif, USA, June 2004.

[18] P. W. Hannan, "The element-gain paradox for a phased-array antenna," IEEE Transactions on Antennas and Propagation, vol. 12, no. 4, pp. 423-433, 1964.

[19] D. F. Kelley and W. L. Stutzman, "Array antenna pattern modeling methods that include mutual coupling effects," IEEE Transactions on Antennas and Propagation, vol. 41, no. 12, pp. 1625-1632, 1993.

[20] D. M. Pozar, "The active element pattern," IEEE Transactions on Antennas and Propagation, vol. 42, no. 8, pp. 1176-1178, 1994.

[21] D. M. Pozar, "A relation between the active input impedance and the active element pattern of a phased array," IEEE Transactions on Antennas and Propagation, vol. 51, no. 9, pp. 2486-2489, 2003. 

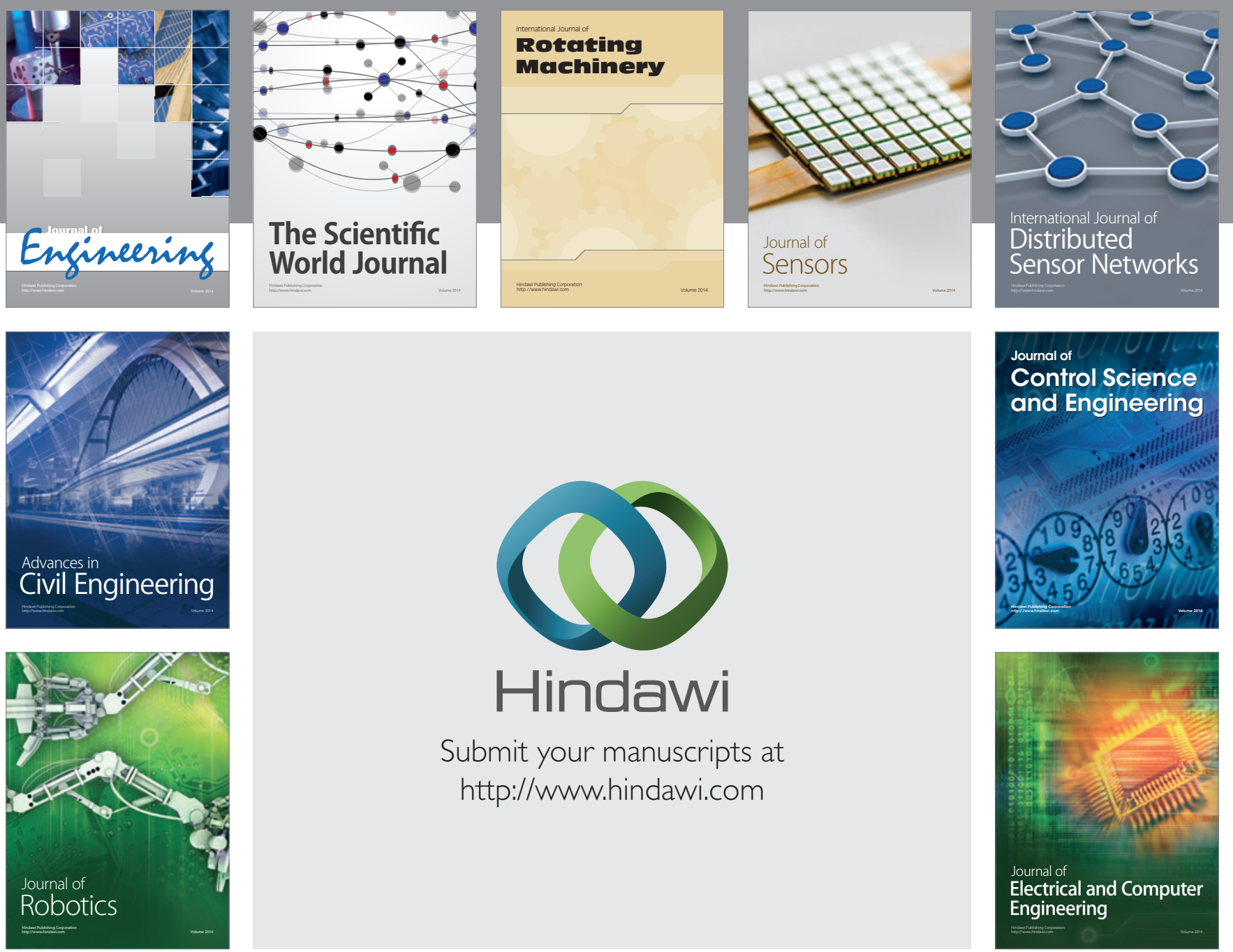

Submit your manuscripts at

http://www.hindawi.com
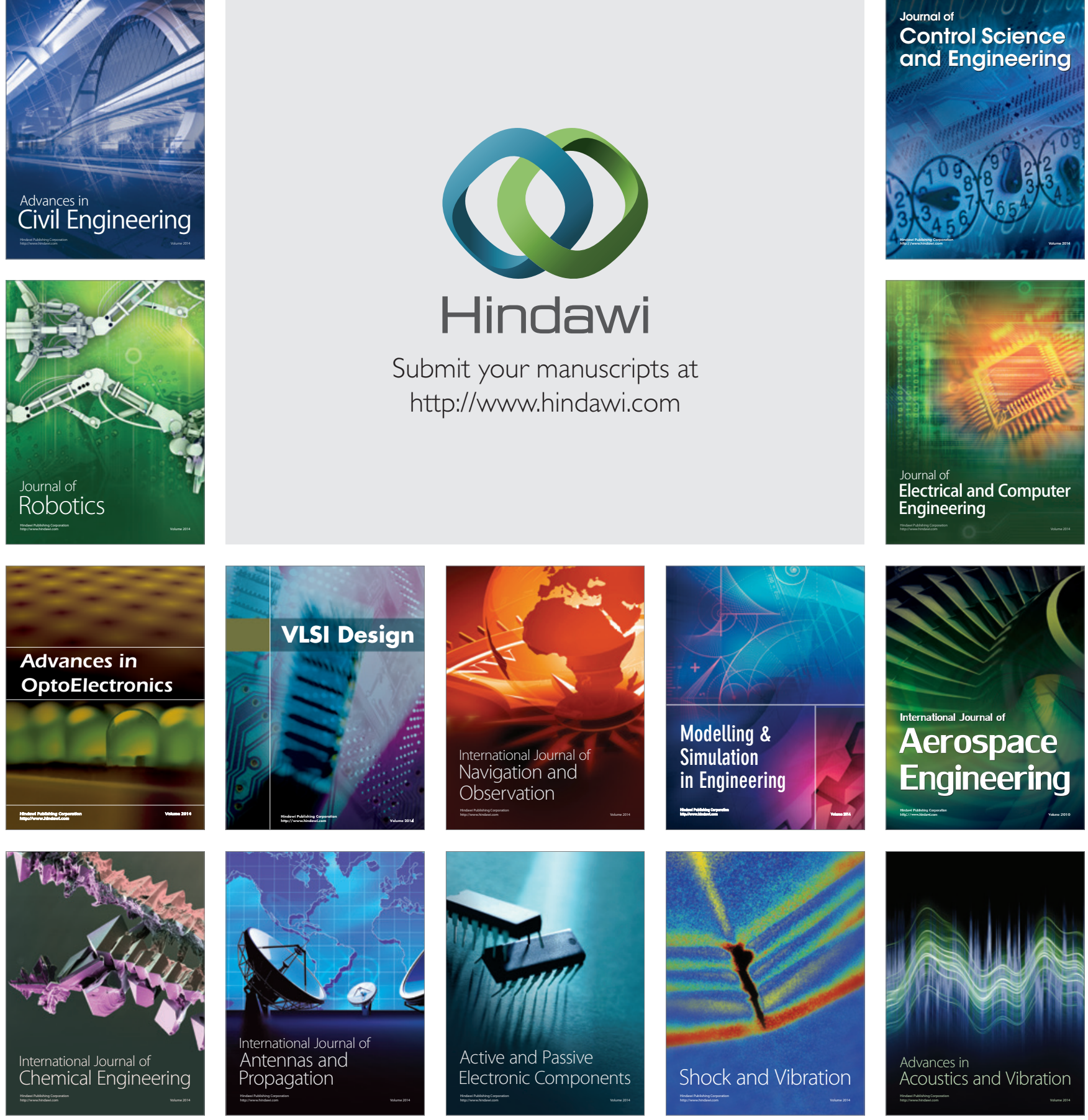